\title{
通过质子耦合电子转移光合成膦酰化氮杂环
}

\author{
易荣楠 ${ }^{a}$ 何卫民*,b \\ ( ${ }^{a}$ 湖南警察学院刑事科学技术系 长沙 410138) \\ ( $b$ 南华大学化学化工学院 湖南衡阳 421001)
}

\section{Proton-Coupled Electron Transfer for Photosynthesis of Phosphorylated $\mathrm{N}$-Heteroaromatics}

\author{
Yi, Rongnan ${ }^{a} \quad$ He, Weimin ${ }^{*, b}$
}

$\left({ }^{a}\right.$ Criminal Technology Department, Hunan Police Academy, Changsha 410138)

$\left({ }^{b}\right.$ School of Chemistry and Chemical Engineering, University of South China, Hengyang, Hunan 421001)

有机磷化合物在合成化学、生物医药、材料科学等 方面具有广泛应用, 因此, 高效构建 $\mathrm{C}-\mathrm{P}$ 键的是有机 合成领域的研究热点 ${ }^{[1]}$. 近年来, 可见光促进的有机合 成方法由于条件温和、绿色高效等优点引起了人们的广 泛关注 ${ }^{[2]}$. 特别值得注意的是, 基于光致氧化还原策略 的磷自由基方法学因其绿色、温和、高效等优点而备受 关注, 相关研究取得较大进展. 2013 年, Kobayashi 课题 组 ${ }^{[3]}$ 报道了小分子有机光敏剂罗丹明 B 催化的烯烃膦酰 化反应, 并提出了基于单电子转移(single electron transfer, SET)和质子转移( proton transfer, PT) 分步历程的磷
自由基引发方式(图 1a). 随后, 吴骊珠课题组 ${ }^{[4]}$ 和吴杰 课题组 ${ }^{[5]}$ 独立发展了钴弜配合物以及曙红 Y 催化的氢原 子转移(hydrogen atom transfer, HAT)过程, 并以此引发 磷自由基串联反应(图 1b). 近日, 郑州大学化学学院元 素化学研究所陈晓岗/於兵课题组 ${ }^{[6]}$ 开发了一种新型咔 唑基二腈苯类光敏剂 $4 \mathrm{CzIPN}-{ }^{-} \mathrm{Bu}$ ，并通过与碱的协同作 用首次实现质子耦合电子转移(proton-coupled electron transfer, PCET)的磷自由基引发方式(图 1c), 并通过后续 的异腈参与的自由基串联环化反应成功构建一系列膦 酰化含氮杂环化合物.

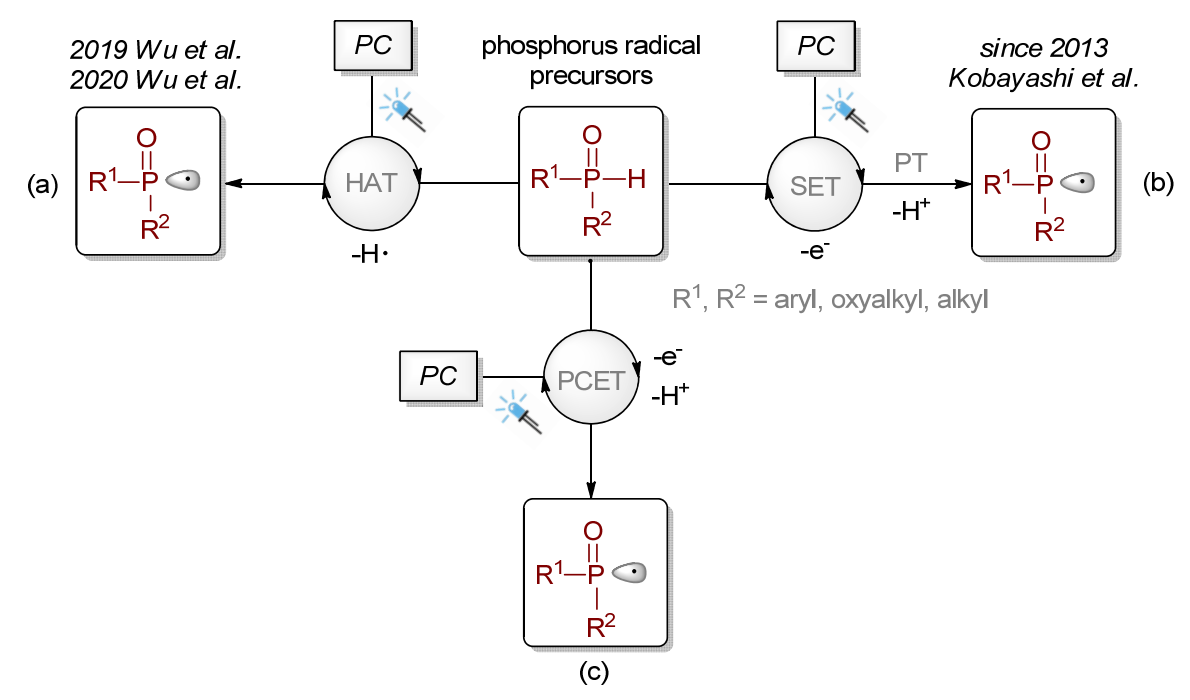

图 1 通过光致氧化还原策略引发磷中心自由基

Scheme 1 Generation of phosphorus-centered radical though photoredox strategy

* Corresponding author. E-mail: weiminhe2016@yeah.net. published online February 23, 2021. 
目前，文献报道的引发磷中心自由基所使用的有机 小分子光敏剂多为基于葱或杂葱结构的苂光染料(如罗 丹明 $\mathrm{B}$ 、曙红 $\mathrm{Y}$ 等), 而陈晓岗/於兵课题组在该反应中 使用的为咔唑基二腈苯 $(\mathrm{CDCB})$ 类光敏剂, 原因在于这 类苂光团具有较好的结构可修饰性以及 HOMO-LOMO 轨道可调节性. 通过对系列 CDCB 类光敏剂进行篎选, 最终确定 $4 \mathrm{CzIPN}-{ }^{t} \mathrm{Bu}$ 为最佳光敏剂. 随后, 将 2-芳基苯 异腈、烯基异腈、2-硫甲基苯异腈用于 $4 \mathrm{CzIPN}-{ }^{{ }} \mathrm{Bu}$ 催化 的自由基串联环化反应，能够成功合成大量膦酰化菲 啶、异喹啉、苯并噻唑衍生物, 并且该反应对于异腈底 物中的各类取代基也具有良好的兼容性(Scheme 2).

值得一提的是，通过比较不同类型磷自由基前 体一一二苯基膦氧、苯基膦酸乙酯、亚磷酸二乙酯的氧 化电位以及 $\mathrm{P}-\mathrm{H}$ 键理论解离能, 阐明了底物在光催化
反应中的反应活性顺序. 经过一系列控制实验，提出了 磷自由基的 PCET 协同引发机制，密度泛函理论(DFT) 计算表明, PCET 历程相比于 SET/PT 或 PT/SET 的分步 过程在反应热力学中更具优势, 从而证实了 PCET 历程 为优势路径. 该研究将有利于推动并完善光致氧化还原 策略引发磷自由基的机制研究.

综上所述，陈晓岗/於兵课题组利用咔唑基二腈苯 类化合物 $4 \mathrm{CzIPN}-{ }^{t} \mathrm{Bu}$ 为光催化剂, 通过与碱的协同作 用实现了质子耦合电子转移作用引发磷中心自由基，与 系列异腈化合物发生自由基串联环化反应成功构建多 种膦酰化含氮杂环化合物. 该反应条件温和, 底物适用 范围较宽，合成的膦酰化菲啶、异喹啉、苯并噻唑等化 合物多达 56 个.

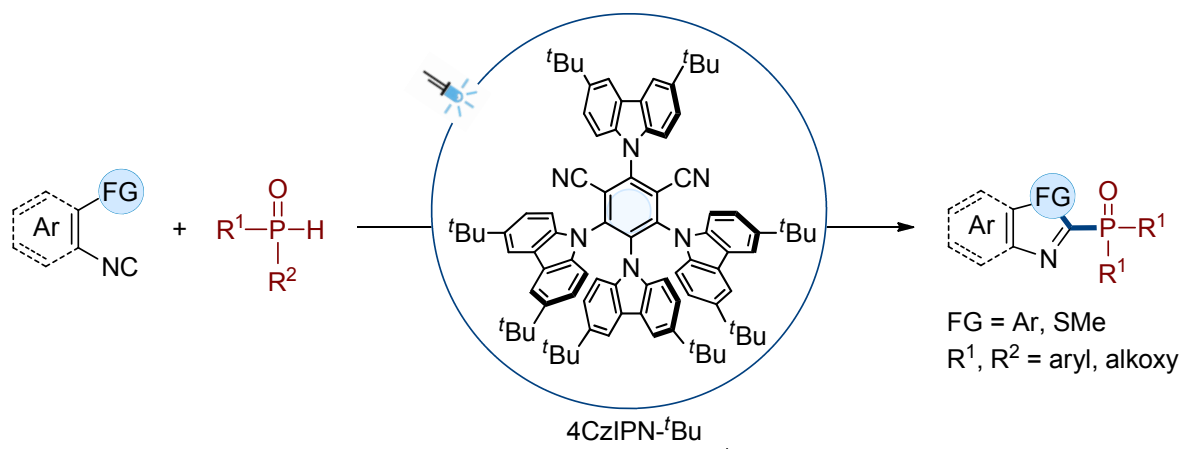

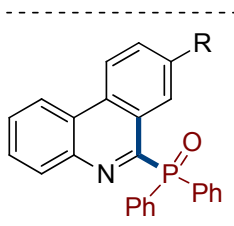

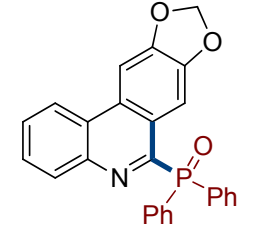

$\mathrm{R}=\mathrm{H}, \quad 73 \%$

$\mathrm{R}=\mathrm{Me}, \quad 64 \%$

$\mathrm{R}=\mathrm{OMe}, 80 \%$

$\mathrm{R}=\mathrm{F}, \quad 75 \%$

$\mathrm{R}=\mathrm{Cl}, \quad 60 \%$

$\mathrm{R}=\mathrm{Br}, \quad 69 \%$

$\mathrm{R}=\mathrm{CF}_{3}, \quad 38 \%$

$\mathrm{R}=\mathrm{CN}, \quad 44 \%$

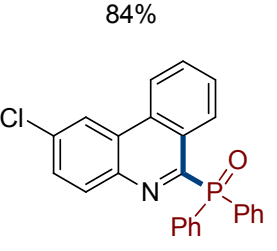

$62 \%$<smiles></smiles>

$46 \%$<smiles>Cc1ccc2c(c1)nc(P(=O)(c1ccccc1)c1ccccc1)c1ccccc12</smiles>

$70 \%$<smiles>O=P(c1ccccc1)(c1ccccc1)c1cc2ccccc2c2ccccc12</smiles>

$55 \%$

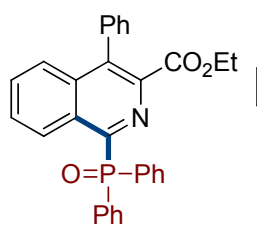

$62 \%$<smiles>Cc1ccc2nc(P(=O)(c3ccccc3)c3ccccc3)c3ccccc3c2c1</smiles>

$49 \%$

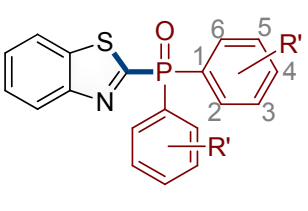

$\mathrm{R}^{\prime}=4-\mathrm{Me}, \quad 90 \%$

$\mathrm{R}^{\prime}=4-\mathrm{OMe}, \quad 53 \%$

$\mathrm{R}^{\prime}=4-\mathrm{F}, \quad 91 \%$

$\mathrm{R}^{\prime}=4-\mathrm{Cl}, \quad 75 \%$ $\mathrm{R}^{\prime}=3,5-\mathrm{Me}_{2} 78 \%$

图式 $2 \mathrm{CDCB}$ 型光敏剂以及底物适用范围

Scheme 2 CDCB-based photocatalyst and substrate scope

\section{References}

[1] (a) Ruan, L.; Liu, C.; Sun, J.; Zhou, M. Chin. J. Org. Chem. 2019, 39, 2403 (in Chinese).

(阮利衡, 刘畅, 孙京, 周明东, 有机化学, 2019, 39, 2403.)

(b) Shi, J.; Wei, W. Chin. J. Org. Chem. 2020, 40, 2170 (in Chinese).

(时建伟, 魏伟, 有机化学, 2020, 40, 2170.)

[2] (a) Sun, K.; Lv, Q.-Y.; Chen, X.-L.; Qu, L.-B.; Yu, B. Green Chem. 2021, 23, 232.

(b) Chen, J.-Y.; Zhong, C.-T.; Gui, Q.-W.; Zhou, Y.-M.; Fang, Y.-Y.; Liu, K.-J.; Lin, Y.-W.; Cao, Z.; He, W.-M. Chin. Chem. Lett. 2021, 32,475 . (c) Liu, K.-J.; Wang, Z.; Lu, L.-H.; Chen, J.-Y.; Zeng, F.; Lin, Y.-W.; Cao, Z.; Yu, X.; He, W.-M. Green Chem. 2021, 23, 496.

(d) Xie, L.-Y.; Peng, S.; Yang, L.-H.; Peng, C.; Lin, Y.-W.; Yu, X.; Cao, Z.; Peng, Y.-Y.; He, W.-M. Green Chem. 2021, 23, 374.

[3] Yoo, W.-J.; Kobayashi, S. Green Chem. 2013, 15, 1844.

[4] Liu, W.-Q.; Lei, T.; Zhou, S.; Yang, X.-L.; Li, J.; Chen, B.; Sivaguru, J.; Tung, C.-H.; Wu, L.-Z. J. Am. Chem. Soc. 2019, 141, 13941.

[5] Yan, J.; Cheo, H. W.; Teo, W. K.; Shi, X.; Wu, H.; Idres, S. B.; Deng, L.-W.; Wu, J. J. Am. Chem. Soc. 2020, 142, 11357.

[6] Liu, Y.; Chen, X.-L.; Li, X.-Y.; Zhu, S.-S.; Li, S.-J.; Song, Y.; Qu, L.-B.; Yu, B. J. Am. Chem. Soc. 2021, 143, 964.

(Cheng, F.) 